\title{
Natorps Einführung in den Idealismus durch Platos Ideenlehre. )
}

\author{
Von A. Görland.
}

Vor beinahe drei Jahren erschien Natorps Werk. Bei seinen 472 Seiten stellt es sich als eingehendste Arbeit über das Gesamtgebiet der Ideenlehre Platos dar. Mit einer kurzen Einleitung beginnend, führt es sogleich über in die Besprechung der Apologie, des Krito, Protagoras, Laches und Charmides (- pag. 28). Im Meno beginnt dann das Wirken des gewaltigen philosophischen Genius. Gorgias, Phaedrus und der bedeutsame Theaetet folgen (- pag. 116). Euthydem, Kratylus, Phaedo, Gastmahl und Strat (- pag. 215) zeigen den Weg zur ersten Gipfelhöhe des platonischen Denkens: zum Parmenides (- pag. 271); ein kurzes Ausruhen im Sophist ( - pag. 296), dann ist die Höhe des Platonismus im Philebus erreicht (- pag. 331). Dem Staatsmann folgt der Timaeus (- pag. 358), der am Ende der platonischen Gedankenarbeit das erkenntniskritische Problem der reinen Raumanschauung entdeckt. Die Gesetze beschliessen die Reihe der Dialoge (- pag. 366). Innerhalb der philologischen Kreise wird diese Abfolge mannigfache Erörterungen noch hervorrufen, weil aus dem philosophischen Sachkriterium der Problemhöhe innerhalb der einzelnen Dialoge auch das zeitliche Nacheinander derselben konstruiert wird. Dies philologische Interesse soll uns aber auf den folgenden Zeilen nicht massgeblich sein. Wir wollen sehen, wie der Erkenntniskritiker Natorp die Genesis des Urerkenntniskritikers Plato zeichnet, um ihm dann zu folgen in seine beiden Schlusskapitel (- pag. 43') "Aristoteles und Plato" und "die aristotelische Kritik der Ideenlehre", in denen die Unzulänglichkeit des Dogmatismus, das Problem und den Arbeitsweg des "methodischen Idealismus" zu begreifen, an jener weltgeschichtlichen Befehdung des Plato durch Aristoteles zur lichtvollen Kennzeichnung gelangt.

Das Werk will die Rechtfertigung der Ansicht sein, dass der platonische Idealismus den strengen Begriff "der Philosophie" inauguriert. Dazu ist der Nachweis nötig, dass die „Ideen" nicht „Dinge", sondern Gesetze bedeuten. Dieser Grundansicht sind ätere Forscher zwar nahe gekommen, aber ihr zumeist durch die aristotelische Autorität wieder abIdealismus.

1) Natorp, Paul, Platons Ideenlehre. Eine Einfuhrung in den 
spenstig gemacht. Erst die Wiedergeburt des Kantischen Idealismus hat zugleich für den Idealismus Platos volles Verständnis gezeitigt. „Ich stehe nicht an, Hermann Cohen als den zu nennen, der uns wie für Kant, so für Plato die Augen geöffnet hat."

Man kann den Meno als Beginn der grossen Entwickelung Platos setzen, vor allem für die Ideenlehre. Er bringt die Entscheidung über das, die früheren Dialoge zusammenhaltende Problem der Lehrbarkeit der Tugend. Der Meno beantwortet die Frage, indem er das „Lernen“ als Wiedererinnern, als ein Schöpfen und Hervorheben der Erkenntnis aus dem erzeugenden Quell des eigenen Bewusstseins vermittels des dialektischen Verfahrens versteht. Es ist eine kühne Wendung des Problems, dass "die Wahjheit von allem, was ist", uns ursprünglich in der Seele, im Bewusstseinsgrunde liege und durch das Verfahren der Frage und der Besinnung daraus hervorzulocken sei. Was ist „das, was ist"? Nicht die einzelnen Thatsachen der Wirklichkeitszufälligkeit, sondern die "reinen" Erkenntnisse der Wissenschaften. Diese sollen somit die reinen Entwickelungen aus den Grundverfahrungsweisen des Denkens selbst sein; denn diese "reinen" Erkenntnisse sind nur die Rechenschaftsberichte des Erkennens über das eigene Gesetz seines Verfahrens. Somit ist eine solche Erkenntnis des Selbst zugleich die Erkenntnis des Objektes, des reinen, d. h. als Gesetz reinen Objekts. Von solcher Art sind die Objekte der Mathematik. Somit bedeutet der Meno die Entdeckung des apriori.

Der Thea etet ist in seinem wichtigsten Teil die für alle Zeiten grundlegende Kritik der Sinnlichkeit. Die Sinnlichkeit ist nur Veranlasser, sie stellt das Problem der Erkenntnis, in dessen Bearbeitung das sich auf sich selbst besinnende Bewusstsein aus der zu bestimmenden - Materie des Sinnes $=\mathrm{X}$ kraft des Begriffs zur bestimmten Erkenntnis, zur Einheit der Prädikation gelangt. Das Sinnliche ist $=X$, denn es ist von grenzenloser Relativität und Variabilität. Die Prädikationen, durch die aus dem sinnlichen Erlebnis Sein, Wahrheit, Erkenntnis wird, vertiefen sich zu Grundprädikationen, zu Kategorien, deren Ausreifung zu Grundideen der $\mathrm{Phaed} o$ bringt.

Der folgenreiche Fortschritt dieses Dialogs liegt in der Anerkennung der Sinnlichkeit als einer Gattung des Seins, neben dem ewigen, unwandelbaren Sein der Ideen. Auf diese muss demnach das erstere Seins bezogen werden. Diese Beziehung stellt das Urteil her. Es muss somit das empirische Urteil in den reinen oder Grundurteilen begriindet sein. Dies Prinzip, die Wahrheit des Empirischen im Logischen zu gründen, in den Setzungen und Grundlegungen des Denkens, ist das Prinzip des Idealismus im pragnanten Sinne des kritischen, bezw. des methodischen Idealismus. Auf diese, nur ihrem obersten

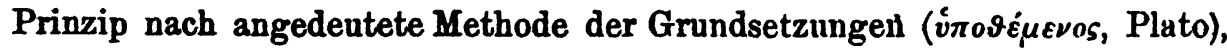
wird der Sinn der Idee restlos zurückgef ührt. Ideen sind nichts als solche Grund-Setzungen ( ${ }^{H}$ Hypothesen ") des Denkens, zum Zwecke der Erkenutnisbewaltigung des Sinnlichen. Etwas ist "schön“", wenn es die Bedingungen erfüllt, dass das aus der Definition resultierende Pră. dikat ( ${ }^{\text {schön }}{ }^{4}$ ) auf es $=X$ als Subjekt beziehbar wird. Dann hat es 
"teil" an der Idee. Dieser von Aristoteles gedchtete Turminus bedentet also nichts als den $\mathrm{E}$ inzelfall des Gesetzes, die Subsumtion (inesse subjecto, Leibnir). Es erbellt, wie wenig Aristoteles seinen Lehrer verstand, als er dus "Teillaben ${ }^{\mu}\left(\mu \dot{\varepsilon} \theta \xi \iota_{5}\right)$ als leere Metapher bczeichnete und tlberzeugt verkundete, dass die Ideen far die övrc nichts leisteten. Vielmelur erkennen wir, dass Plato genau das einsieht, was Kant ausspricht: dass wir von den Gegenständen nur das begreifen, was wir zuvor, unseren Begriffen, unseren (Wissenschafts-)Hypothesen zufolge selbst in sie hineingelegt haben.

An der Schwelle des bedentsamen Parmenides überschanen wir folgende Ergebnisse: Ideen bedeuten nicht Dinge, sondern Methoden. Als solche reinen Setzungen des Denkens sind sie auf den unbeschränkten Fortgang eines Verfahrens angewiesen, dessen von Stufe zu Stufe gewonnene Hrgebnisse allenfalls hypothetische, nie: absolute Gegenstïnde heissen kornnen; es sind vielmehr Lösungen von Problemen, die stets neue Probleme zu tage fördern, neue Lösungen verlangen. Dadurch sind Ideen: Grundsătze des Denkens zum Zwecke der Erforschung der Phaenomene. Diese „haben Teil“ an ihnen, d. h. sie sind als stufenmässige Auswickelung der Verfahrungsweisen, welche die Ideen bedeuten, zu denken, wenn auch nicht darzustellen. Denn diese „stufenmässige Entwickelung " ist ein unendlicher Weg, ist (unendliche) Aufgabe. Somit sagt "Idee ${ }^{\text {das }} \mathrm{Ziel}$, den unendlich fernen Punkt, der die Richtung, die der Weg der Erfahrung nimmt, bestimmt.

Die Wissenschaft der Ideen heisst Dialektik. Je reiner die Dialektik von Plato hervorgehoben wurde, um so deutlicher entstand die Gefahr, dass die Idee von der Aufgabe, Erfahrung als Wissenschaft zu begründen, losgelöst werde. Wer nicht in den Ideen die Methodenbedeutung, die Erkenntnisaufgabe gerade für die Dinge erkannte, dem mussten sich die Ideen an die Stelle der abgelehnten „Dinge ${ }^{\alpha}$ der vulgären Erfahrung setzen.

Diese Gefahr abzuwenden, scheint der Parmenides gewidmet zu sein. Sind die Grundbegriffe von den Phaenomenen rein zu sondern? Sind sie also etwa nur eine Art zweiter Ordnung von ${ }_{n}$ Dingen ${ }^{4}$, hinter, neben oder über den Sinnendingen? Die Angriffe, die Plato im „Parmenides“ gegen eine so „verstandene ${ }^{\text {“ }}$ Ideenlehre richtete, sind erbarmungslos.

Im Dialog wird der junge Sokrates durch den alten Parmenides von der absurd dinglichen Auffassung der "Metapher" des "Teilhaben" hefreit. Das einzelne Schöne hat teil an der Idee des Schönen, nicht „dem Teile ${ }^{*}$ oder „dem Ganzen nach“, sondern wie das Einzelne unter der Einheit der Idee befassbar ist; denn „Einheit der Idee“ bedeutet „Einheit des Bewusstseins". Es ist die "Einheit", die uns entsteht, indem wir; auf das Viele hinsehend, es in dieser bestimmten "Hinsicht" als ein Bestimmtes, z. B. „Schön ${ }^{*}$ erkennen. So ersehen wir die Idee mit der Seele.

Auch nach der Ablehnung eines psychologischen Idealismus und nach der Behauptung eines methodischen Idealismus, der keinerlei „Existenz denkender Bewusstseine" vorauszusetzen gezwungen ist, $v \in r-$ bleibt gleichwohl die wuchtigste Schwierigkeit für die Ideenlehre noch: 
Wie ist das Reich der Erfahrung in der ganzen Unendlichkeit ihrer Relativität der Methode der Ideen zu unterwerfen? Wie haben wir überhaupt die reinen Grundbegriffe? Haben wir sie, so stehen sie im Charakter der empirischen Gegenstände; denn diese sind uns allein „gegeben“. Da wir aber nur Erfahrung hahen, können die Ideen als "reine“, als apriori Begriffe - Gegenstände der Erfahruug sein?

Hier giebt die Klärung der Hinweis auf das Verfahren der Deduktion. Sie ist der Weg der Vergewisserung der Grundbegriffe. Das Gelingen der Deduktion ist die einzig mögliche, aber auch zureichende Probe, dass wir die rechten Grúndbegriffe haben. Insofern wäre es nicht widersinnig, von einer empirischen Kenntnis der apriori-Erkenntnis zu sprechen. Wir erreichen mit dem apriori den Gegenstand der Erfahrung, weil die Idee die Grundlage der Möglichkeit, d. i. die methodische Begründung der Erfahrung ist; Deduktion ist das Herauslesen dessen, was wir zuvörderst in die Gegenstände gelegt haben. Dieser transscendentale Idealismus ist die neue Ahnung Platos.

Wie sehr nun der Erfahrungsgegenstand ein unendlicher ist, so ist. es doch möglich, mit dem Verfahren der Deduktion ihn zu umspannen, weil es sich bei aller Unendlichkeit der Erfahrungswelt doch nur um ein geschlossenes System reiner Begriffe handelt. In der Grösse des Entwurfs desselben liegt die Bedeutung des Parmenides. Plato nimmt den Begriff des „Einen" zum Ausgangspunkt und fasst die Einheit als einen Ausdruck der Denkfunktion überhaupt. Das Subjekt der Aussage, das $\mathrm{X}$ der Bestimmung, ist alsdann das "Nicht-Eine ${ }^{\text {i }}$ oder „das Andere $^{\mu}$. Dies ist das Gebiet der Relativität; alle Setzung der reinen Denkfunktionen gelten nur in bestimmter Bezüglichkeit, und die Gesamtheit aller bezüglichen Setzungen der reinen Begriffe ist der Erfahrungsgegenstand $=X$. Damit ist die Möglichkeit der Erfahrung erwiesen, kraft der reinen Setzungen des Denkens. Denn das Subjekt unserer Erkenntnisaussage, das Erfahrungs $=\mathrm{X}$, ist jenen ge mäss.

Weil alle Bestimmung des Erfahrungsgegenstandes in unendlicher Relativität der Denksetzungen verläuft, es also keine absoluten Bestimmungen giebt, so sind die "Dinge" nur Probleme. Die reinen Begriffe, die „Ideen“ aber können in die Beziehung zu einander treten, weil es Funktionen des Bewusstseins, und nicht - transscendente Dinge sind. -

Der Grund aller dialektischen Schwierigkeiten ist der, dass Einheit und Vielheit sich in den Sinnendingen, - weil in den Ideen findet. Denn kraft des logischen Sinnes der "Aussage" ist Dasselbe sowohl ein Eines wie ein Vieles. Das ist das unsterbliche Begegnis des Logischen selbst in uns.

Die Aussage der „Einheit" und „Vielheit" schliesst ursprïnglich ein die „Bestimmung" und die "Unbestimmtheit". Darum miisse man, sagt der Philebus, in Hinsicht des ganzen Gebietes jedes jeweiligen Problems erstlich die Einheit setzen (die Idee) und unter ihrer Voranssetznng die l'ntersnchnng füluren. : Dann müsse man nach weiteren Ideen suchen; dann werde man endlich finden, dass die ursprüngliche 
Einheit sich in eine unendliche Vielheit von Ideen vertieft (Idee des Unbestimmten).

Somit ist alle Aussage die Einheit verschiedener, aber sich verflechtender begrifflicher Bestimmungen. Und darin wurzelt die Idee. Die Einheit des Ausgesagten ist die Einheit des Mannigfaltigen. Darin haben wir den lauteren wie schlichten Ausdruck des idealistischen Grandprinzips der Philosophie Platons: Dass von dem logischen Sinn der Aussage, als dem einzig möglichen Anfang, auszugehen ist und, was als Bedingung der Moglichkeit der Aussage überhaupt, somit als unzerstörbarer Charakter des "Logischen in uns" sich erweist, schlechterdings zu Grunde zu legen ist, als Fundament aller weiteren Aufstellungen der Erkenntnis.

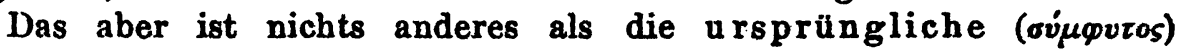
Korrelation des Unbestimmten und seiner Bestimmung, des $X$ zum A der Erkenntnis. Das wird als letzter und erschópfender Sinn des Verfahrens der Idee erklart. Einheit der Idee ist nichts anderes als Einheit der Bestimmung und zwar jenes Unbestimmten, welches im Parmenides unter dem Terminus a $\pi \varepsilon \varepsilon \rho i \alpha$ als Korrelatbegriff zum eidos entdeckt wurde.

Fast Kantisch lautet es, dass man in jedem Problemgebiete eine solche Einheit setzen und unter ihrer Voraussetzung dann nntersuch en müsse, so werde man sie, die man selber gesetzt hatte, auch darin finden.

Den Übergang von der Einheit auf die unendliche Mannigfaltigkeit, Unbestimmtheit erklärt Plato nicht als Sprung, sondern als kontinuier$l_{i}$ ch en Fortgang der Determination ins Besondere, als eine unendliche Spezifikation, um sich dem letzten Problem unserer Erkenntnis, dem Erfahrungsgegenstand $=\mathrm{X}$ möglichst zu nähern. Hat die Spezifikation vom allgemeinen Gesetze zum besonderen eine solche Grenze erreicht, dass eine weitere Besonderung keine neue Gesetzeserkenntnis mehr erreicht, so hat die weitere Zerlegung in Einzelfälle kein wissenschaftliches Interesse mehr.

Diese Gedanken sind höchst bezeichnend für die empirisch-wissenschaftliche Richtung; die jetzt die Ideenlehre genommen hat, dass geradezu als Unterscheidungsmittel des Dialektischen vom eristischen Verfahren festgesetyt wird: Nicht bei dem generellen, im schlechten Sinne bloss "logischen" Verhältnis der Einheit des Gesetzes und der unendlichen Unbestimmtbeit des Empirischen stehen zu bleiben (z. B. bei dem allgemeinen, bloss schematischen Verhältnis von Ursache und Wirkung), sondern die $Z$ wischenglieder in Vollständigkeit aufusuchen, d. h. die besonderen Gesetze zu erforschen, die dem allgemein logischen Verhältnis des Gesetzes zum Einzelvorkommnis erst den bestimmten wissenschaftlichen Sinn geben. Im philosophischen Interesse ist hierbei aber zu beachten, dass die Entfaltung des Empirischen gerade aus der logischen Natur der Aussage, aus dem Gesetz des Urteils selbst hergeleitet wird, dass aber das sogenannte „empirische ${ }^{u}$ Verfahren erkannt ist als das konkret Logische. -

Am Ende der gewaltigen Entwickelnng der Philosophic Platous, dic nun aus dem Dialogganzen konstruiert ist, vermag der Philosophiehisto- 
riker der Gerechtigkeit zu walten; die bislang durch die Jahrhunderte gefehlt hat, wo es galt, den einer gemeinverständlichen Gedankenrichtung gefalligen Aristoteles in die mit wachsender Kraft wissenschaftlicher Naturerkenntnis unaufhaltsam sich verengenden Schranken zurückzuweisen zum Heile der bislang mehr poetisch goutierten als wissenschaftlich ernst genommenen "Ideenlehre". Platons.

So wahr Dogmatismus und methodischer Idealismus unversöhnlicheWidersacher sind, so wahr ist ein Aristoteles ewig unfähig, sich in den Platonischen Gesichtspunkte zu versetzen.

Erkenntnis und Gegenstand sind gewisslich Korrelation, wie Weg und Ziel, das man vor Augen haben muss, um den Weg zu gehen. Den Begriff des „Gegenstandes“, der erkannt werden soll, als das X der Gleichung der Erkenntnis, setzt aber freilich voraus, wer von Erkenntnis überhanpt mit klarem Sinn spricht. Aber der Kritizismus betont, dass es nur ein X, dass der Gegenstand stets Problem, nie Datum ist; ein Problem, dessen ganzer Sinn bestimmt ist allein in Beziehung auf die bekannten Grössen der Gleichung, nämlich unsere fundamentalen Begriffe, die nur die Grundfunktionen der Erkenntnis selbst, die Gesetze des Verfahrens, in dem Erkenntnis besteht, zum Inhalte haben. Diese aber könnten wir eher - der Erkenntnis "gegeben" heissen, sofern mit ihnen und durch sie erst eine Erkenntnis möglich wird. Der Gegenstand aber ist nicht ein Datum, sondern Problem, dessen Bestimmungs-Bewältigung ein prinzipiell unendlicher Weg ist. Es deckt sich sonach die kritische Ansicht mit der genetischen.

Thr steht die Ansicht gegenüber, dass der Gegenstand durch Sinneswahrnehmung wesentlich und ursprünglich für die Erkenntnis gegeben sein müsse, wenn über ihn „objektiv" solle etwas ausgemacht werden können. Der Gegenstand und seine Erkenntnis decken sich, dem Inhalte nach, nur dass seine Bestandteile im ursprünglich Gegebenen in ungeschiedener Verflechtung, in der reifen Erkenntnis deutlich auseinander gestellt und einzeln zum Bewusstsein gebracht sind. Somit verhält sich Gegenstand zur Erkenntnis wie Konkretes zu Abstraktem. Das Konkrete geht vorher und bezeichnet die Aufgabe für die Erkenntnis. Also deckt sich die dogmatische Ansicht der Erkenntnis mit der abstraktiven.

Für den Dogmatist ist der Gegenstand gegeben, ist "da"; dann sind auch die Faktoren gegeben, deren Produkt der Gegenstand ist. Es kommt nur darauf an, diesen impliciten Besitz gewusst-explicit zu machen.

Der Kritizist betrachtet die Determination des „Gegenstandes ${ }^{6}=X$ der Erkenntnis als eine unendliche, obzwar geforderte. Alle (erreichte) Bestimmtheit der Erkenntnis im Problembegriff des Gegenstandes ist nur Năherungswert. Deshalb nimmt der Kritizismus seinen Standpunkt grundsätzlich im Wege (der Erkenntnis) und nicht im Ziel (Gegenstand); er sagt, dass von der Erkenntnis aus das „Sein ${ }^{4}$, nämlich das "Sein" der Erkenntnis, nicht vom ${ }_{n}$ Sein $^{\mu}$ aus, als hatten wir es, die Erkenntnis ver. ständlich zu machen sei.

Für den Dogmatisten ist der Gegenstand $\eta^{\text {an }}$ sich $^{\alpha}$, was er ist, nicht bloss von Gnaden der Erkenntnis. 
Indem Plato die Erkenntnis als Bestimmung eines Unbestimmten, eines unendlich Bestimmbaren, als Begrenzung cines in sich Grenzenlosen, alser ohne Sohranke fortsclireitender Begrenzung Fahigen behauptet, spricht er den Sinn des Kritizismus in kaum zu uberbietender Bestimmtheit hus.

Ebenso entschieden behauptet Aristoteles die abstraktive Ansicht. Von ihr aus, als der selbstverständlichen, unerschütterlichen Voraussetziung, beurteilt er Plato. Darum muss er ihn in jeder Aufstellung missverstehen. Wie abe: kann man unter solchen Cmständen fortfahren, Aristoteles als den berufenen Nachfolger Platos, als den natürlichen Erben und mächtigen Fortbildner seiner Philosophie darstellen? Dagegen würden gleich entschieden beide Philosophen Verwahrung einlegen.

Durch die Logik und Psychologie und entscheidend durch die Metaphysik des Aristoteles führt Natorp die Kontroverse des Aristoteles zu Plato hindurch, als Kontroverse des Dogmatisten gegen den Kriticisten.

Fordert Aristoteles eine deduktive Begründung der Erkenntnis aus

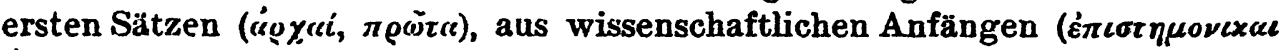
‘ $\rho \chi(c i)$, so bedeutet es nicht die Anerkennung der. Erzeugung des Gegenstandes aus den Bedingungen der Erkenntnis, sondern die Anerkennung jenes Rationalismus, dass das Sein gewissen denk-gemässen Bestimmungen unterliege, dem Denken wie ersichtlich - entspreche.

Ist es Platos fundamentale Forderung, den empirischen „Gegenstand als Erkenntnisaufgabe grundsätzlich in eine unendliche Reihe von Bestimmungen funktionellen, nicht dinglichen Charakters aufzulösen, so fordert Aristoteles, dass der Beweis für den Gegenstand beiderseits endlich, abschliessend gegeben werden müsse. Denn der Sinn ist: der Gegenstand ist gegeben, und dieses Datum, das wir haben, hat der Beweis, dem Daseinswerte des Gegenstandes gemäss, abschliessend auszusprechen.

Die letzten Stützen des Beweises, jene „ersten Sätze“, sind aber "durch sich" gewiss, einer logischen Rechtfertigung anbedürftig. Wir kennen darüber das scharfe Verdikt Kants. „Dann wäre alle Kritik des Verstandes verloren“, die ganze Liebesmühe des philosophischen Eros, möchte Plato sagen, umsonst. Die letzte Stütze aller Erkenntnis der Prinzipien ist nicht eine transscendentale Deduktion, wie sie Aristoteles für den Identitätssatz zwar in Angriff nimmt, wenngleich er ihr nicht treu bleibt, sondern die Induktion im vulgären Sinne des „Wir sehen es", „es ist augenscheinlich", „es zeigte sich nichts anderes". Und selbst die Wege der Abstraktion sind von den Sinnen vorgezeichnet.

Der wahre primäre Gegenstand der Erkenntnis ist nicht, wie dem Kritizisten Plato: die Bedingungen ihrer Möglichkeit, sondern für den Dogmatisten Aristoteles die konkrete Einzelsubstanz. Und nun beginnt das dunkle, unentwirrbare Schwanken zwischen dieser, und dem "Allgemeinen“. Hat aber Aristoteles begriffen, dass der Einzelne genau so nur das Einzelne des Allgemeinen ist, wie das Allgemeine das Allgemeine des Einzelnen? Dass das Einzelding nicht "getrennt", ineben* dem Allgemeinen gesetzt werden darf, wie das Allgemeine nicht ${ }_{n}$ neben ${ }^{*}$ und „getrennt ${ }^{\mu}$ vom Einzelding? Das ganze Verbaltnis des Allgemeinen zum 
Einzelnen findet nur in der Erkenntnis statt, ist nur als Gesetz des I. ogi schen zu verstehen, ist die Eigenheit der hóyoc, der Setzungen des Denkens "in uns", welche in dieser unaufheblichen Korrelation ihren Ausdruck findet. Das Einzelne ist nur das Einzelne in ver einzelnder Betrachtung, wie das Allgemeine es ist im allgemeinen Gesichtspunkt des Denkens. Das aber weiss Aristoteles nicht.

Dem dogmatischen Denker sind darum die Kategorien nicht Grundformen des Denkens, sondern Grundformen der Daseinsweisen des Ei nz e 1dinges. Darum sind die beiden Kategorien des Thuns und des Erleidens nicht Eine Relation von Ursache und Wirkung im Kantischen Sinne, sondern zwei Aussageweisen.

Für Kant dagegen lösen sich die Dinge ganz und gar in Verhältnisse auf, in eine Unendlichkeit von Relationen; die Beziehungspunkte einer Relation sind zwar gegeben, aber ganz und gar nur als hypothetische ansetzbar. Somit ist das "Ding“, die unzerstörbar beharrende Existenzgrundlage das Allerletzte, was unserer Erkenntnis zur Aufgabe gestellt ist. Absolute Erkenntnis ist uns unerreichbar.

Hier hat die kritische Arbeit ein für alle mal gegen Aristoteles entschieden dadurch, dass der Substanzsatz, wie jeder Erkenntnissatz, nicht ein analytischer, sondern ein synthetischer Satz ist. Er bedeutet ein Verfahren, den Gegenstand in der Erfahrung erst auf $z$ u buen, bedeutet das Gesetz eines Prozesses der Erkenntnis, der ein unendlicher, abschlussloser nicht nur für das allgemeine Problem, sondern für jeden „Einzelgegenstand “ ist. Und Gegenstand als Substanz ist das, was wir als identischen Beziehungspunkt unserer Aussage mit jederzeit relativer, nie absoluter Gültigkeit ansetzen.

Somit ist das „Nicht-erschöpft-werden" des Einzelfalles durch . die Definition, was Aristoteles bedauert, in schroffstem Gegensatz zu Aristoteles gerade das Wesen aller Erkenntnis. Die Definitionsbeziehung. zwischen $\operatorname{dem} X(S)$ und $\operatorname{dem} A(P)$ ist mit nichten. Identität. Da ist die Wurzel des aristotelischen Irrtums, der allerdings von der Mehrzahl der heutigen Logiker noch geteilt wird. Von solcher dogmatischen, abstraktiren Methode her vermag Aristoteles die genetische Methode Platos nicht zu begreifen. Unter seinen Händen wird die Ideenlehre Platos, anstatt. zum Urtypus des echten methodischen Idealismus, den wir nach Kant Kritizismus zu nennen gewohnt wurden, zu dem wehrlos bespöttelbaren Zerrbild eines mystischen Dogmatismus von Ideendingen, über den sich der empirische - Dogmatismus des Aristoteles erhaben dünkt.

Aber, wie wir zu zeigen versuchten, giebt nicht nur das historische Richteramt, dessen Natorp versieht, dem Buche seine Bedeutung. Der weite Horizont dieses Buches, in dessen Bezirk die Philosophie des Plato gegen die Philosophie des Aristoteles verteidigt, gerechtfertigt wird, erhebt jene Gestalten zu Repräsentanten der beiden Denkrichtungen der Menschheit. So lehrt uns Natorp, aus dem Thesauros Kants die ahnungstiefen Worte Platos zu deuten und unser eigenes Denken zum methodischen Idealismus reif zu machen, indem er uns den geraden Weg des Kritizismus von Plato zu Kant führt. 